\title{
Exfoliation solvent dependent plasmon resonances in two-dimensional sub-stoichiometric molybdenum oxide nanoflakes
}

Alsaif, Manal M Y A; Field, Matthew; Daeneke, Torben; Chrimes, Adam; Zhang, Wei; Carey, Benjamin; Berean, Kyle

https://researchrepository.rmit.edu.au/esploro/outputs/9921860122101341/filesAndLinks?institution=61RMIT_INST\&index=null

Alsaif, M. M. Y. A., Field, M., Daeneke, T., Chrimes, A., Zhang, W., Carey, B., Berean, K., Walia, S., van Embden, J., Zhang, B., Latham, K., Kalantar Zadeh, K., \& Ou, J. (2016). Exfoliation solvent dependent plasmon resonances in two-dimensional sub-stoichiometric molybdenum oxide nanoflakes. ACS Applied Materials and Interfaces, 8(5), 3482-3493. https://doi.org/10.1021/acsami.5b12076 Document Version: Accepted Manuscript

Published Version: https://doi.org/10.1021/acsami.5b12076 
Thank you for downloading this document from the RMIT Research Repository.

The RMIT Research Repository is an open access database showcasing the research outputs of RMIT University researchers.

RMIT Research Repository: http://researchbank.rmit.edu.aul

\section{Citation:}

Alsaif, M, Field, M, Daeneke, T, Chrimes, A, Zhang, W, Carey, B, Berean, K, Walia, S, van Embden, J, Zhang, B, Latham, K, Kalantar Zadeh, K and Ou, J 2016, 'Exfoliation solvent dependent plasmon resonances in two-dimensional sub-stoichiometric molybdenum oxide nanoflakes', ACS Applied Materials and Interfaces, vol. 8, no. 5, pp. 3482-3493.

See this record in the RMIT Research Repository at:

https://researchbank.rmit.edu.au/view/rmit:36379

Version: Accepted Manuscript

Copyright Statement:

(c) 2016 American Chemical Society

Link to Published Version:

https://dx.doi.org/10.1021/acsami.5b12076 


\section{Exfoliation Solvent Dependent Plasmon Resonances in Two Dimensional Sub- Stoichiometric Molybdenum Oxide Nanoflakes}

Manal M. Y. A. Alsaif, ${ }^{, a}$ Matthew R. Field, ${ }^{\mathrm{b}}$ Torben Daeneke, ${ }^{\mathrm{a}}$ Adam F. Chrimes, ${ }^{\mathrm{a}, \mathrm{c}}$ Wei Zhang, ${ }^{\text {a }}$ Benjamin J. Carey, ${ }^{\text {a }}$ Kyle J. Berean, ${ }^{\text {a }}$ Sumeet Walia, ${ }^{d}$ Joel van Embden, ${ }^{\text {e, }}{ }^{\text {f Baoyue }}$ Zhang, ${ }^{\mathrm{g}}$ Kay Latham, ${ }^{\mathrm{e}}$ Kourosh Kalantar-zadeh ${ }^{*, \mathrm{a}}$ and Jian Zhen Ou, ${ }^{*, \mathrm{a}}$

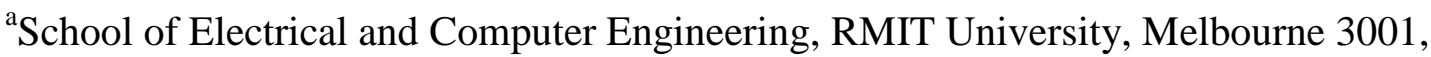
Australia

${ }^{\mathrm{b}}$ RMIT Microscopy and Microanalysis Facility, RMIT University, Melbourne 3001, Australia ${ }^{\mathrm{c}}$ Institute of Chemical and Bioengineering, Department of Chemistry and Applied Biosciences, ETH Zürich, Zürich 8092, Switzerland

${ }^{\mathrm{d}}$ Functional Materials and Microsystems Research Group, RMIT University, Melbourne 3001, Australia

${ }^{\text {e}}$ School of Applied Sciences, RMIT University, Melbourne 3001, Australia ${ }^{\mathrm{f}}$ CSIRO Manufacturing Flagship, Bayview Avenue, Clayton 3168, Australia 'Xi'an Institute of Electromechanical Information Technology, Xi'an 710000, China 
ABSTRACT: Few-layer two dimensional (2D) molybdenum oxide nanoflakes are exfoliated using a grinding assisted liquid phase sonication exfoliation method. The sonication process is carried out in five different mixtures of water with both aprotic and protic solvents. We found that surface energy and solubility of mixtures play important roles in changing the thickness, lateral dimension as well as the synthetic yield of the nanoflakes. We demonstrate an increase in proton intercalation in 2D nanoflakes upon simulated solar light exposure. This results in sub-stoichiometric flakes and a subsequent enhancement in free electron concentrations, producing plasmon resonances. Two plasmon resonance peaks associated with the thickness and the lateral dimension axes are observable in the samples, in which the plasmonic peak positions could be tuned by the choice of the solvent in exfoliating 2D molybdenum oxide. The extinction coefficients of the plasmonic absorption bands of 2D molybdenum oxide nanoflakes in all samples are found to be high $\left(\varepsilon>10^{9} \mathrm{~L} \cdot \mathrm{mol}^{-1} \cdot \mathrm{cm}^{-1}\right)$. It is expected that the tunable plasmon resonances of 2D molybdenum oxide nanoflakes presented in this work can be used in future electronic, optical, and sensing devices.

KEYWORDS: two-dimensional, molybdenum oxide, solar light, plasmon resonance, extinction coefficient, biosensing 


\section{INTRODUCTION}

Plasmons are the collective oscillations of valence electrons in conducting materials, ${ }^{1}$ which provide opportunities to create sensitive measurement tools and new technologies in optics, photo-thermal therapy, chemical sensing and biosensing. ${ }^{2-6}$ The recent demonstration of plasmon resonances in two dimensional (2D) materials have presented several advantages over the conventional noble metal plasmonic systems, in particular the in situ tunability of plasmon resonance peaks. ${ }^{4-8} 2 \mathrm{D}$ materials are planer structures with relatively large aspect ratios (lateral dimension/thickness). ${ }^{9-10}$ These planes are made of covalently bound atoms arranged in layers. ${ }^{11-12}$ If these $2 \mathrm{D}$ materials are made of more than one plane stacked on top of each other, then such planes are held together by weak van der Waals forces. ${ }^{12-13} 2 \mathrm{D}$ materials can show unique electronic and optical properties when the number of planes are reduced, due to changes in the electronic band structure. ${ }^{11}$ Furthermore many $2 \mathrm{D}$ materials, such as graphene and transition metal dichalcogenides, demonstrate a plasmon resonant peak within the mid-infrared and terahertz (THz) range in their intrinsic state. ${ }^{1,7,14}$ The plasmon resonances in these materials are limited to the low energy spectrum due to insufficient free charge carrier concentrations, ${ }^{1-2,}$ 7, 14-16 which limits the practical applications of plasmonics in these types of materials as $\mathrm{THz}$ light sources, detectors and spectrometers are not commonly accessible. ${ }^{15-17}$

Reduction of the material thickness in 2D materials can however lead to alterations in their plasmon dispersion relation. ${ }^{4,13}$ Plasmon dispersion is highly dependent on the geometrical configuration of the nanostructures., ${ }^{5}$ 18-19 Theoretically, the existence of one large depolarization factor in the thickness axis of $2 \mathrm{D}$ materials can be used to tune the plasmon resonance response into the visible and near-infrared (NIR) regions, provided that a sufficiently large free charge carrier concentration is present in the material. ${ }^{4}$ Obtaining plasmon resonances in the visible and NIR regions is an important goal for the development 
of future sensing and plasmonic data processing units, as these wavelengths are commonly used in standard optical systems. ${ }^{4}$ Methodologies including heterostructuring and gating can also effectively tune 2D materials by altering their free charge carrier concentration. ${ }^{20}$ However, plasmon resonant wavelengths responses utilizing these methods are still limited to the mid- and far-infrared regions. ${ }^{1,21}$

Ion intercalation has so far been shown to be the best method to increase the free charge carrier concentrations in 2D material to achieve plasmon resonances in the visible and NIR regions. ${ }^{4}$ We have already shown that both the electrochemical and photochemical methods can be used for achieving intercalation at desired levels in $2 \mathrm{D}$ metal oxides and dichalcogenides. ${ }^{3-4}$ However, the means to gain the desired tunability for NIR and visible light plasmon resonances is yet to be fully investigated.

Free electron concentrations in 2D molybdenum oxide $\left(\mathrm{MoO}_{3}\right)$ can be significantly increased by inducing oxygen vacancies using $\mathrm{H}^{+}$ion intercalation into the interlayer spacing of this material. ${ }^{4,22}$ We previously demonstrated quasi-metallic 2D sub-stoichiometric molybdenum oxide $\left(\mathrm{MoO}_{3-x}\right)$ nanoflakes ( $x$ is the sub-stoichiometric level) synthesized utilizing grinding assisted liquid phase exfoliation (LPE) in water based solvents in the presence of simulated solar light. ${ }^{4}$ We showed the emergence of strong plasmon resonance peaks in the visible range as a result of high sub-stoichiometric levels, ${ }^{4}$ subsequently demonstrating tunability of these plasmon resonance peaks by exposing 2D flakes to different solar light exposure durations. ${ }^{4}$ The electron-hole pairs which are formed upon photo-excitation of $\mathrm{MoO}_{3}$ by simulated solar light and the photogenerated holes oxidize water molecules to produce oxygen and proton entities. ${ }^{22}$ The photo-excited electrons remain in the conduction band (CB), providing the driving force for the $\mathrm{H}^{+}$intercalation into the nanoflakes. ${ }^{22}$ The intercalated $\mathrm{H}^{+}$ions predominantly bind to edge-shared oxygen and terminal oxygen atoms, 
establishing intermediate $\mathrm{OH}_{2}$ groups. ${ }^{23-24}$ These unstable $\mathrm{OH}_{2}$ groups are eventually released from their original positions in the crystal lattice and consequently forming $\mathrm{MoO}_{3-\mathrm{x} .}{ }^{22-24}$ Compounds of $\mathrm{MoO}_{3-x}(0<x \leq 0.125)$, such as $\mathrm{Mog}_{9} \mathrm{O}_{26}$ and $\mathrm{Mo}_{8} \mathrm{O}_{23}$ are $n$-doped semiconductors (free electron concentration of $\sim 10^{20}-10^{21} \mathrm{~cm}^{-3}$ ). ${ }^{22}$ However, other substoichiometries of this compound with higher $x(0.125 \leq x<1)$ show quasi-metallic behavior (free electron concentration of $\sim 10^{22} \mathrm{~cm}^{-3}$ ). ${ }^{22}$

Successful LPE of a stratified material depends on overcoming van der Waals attraction forces between the adjacent layers. ${ }^{9}{ }^{25-26}$ This requires either an initial increase between the interlayer spacing (that occurs through intercalation) $)^{3-4}$ or a solvent with matching surface tension properties. ${ }^{25,} 27$ The LPE process is facilitated by intense ultrasonic waves, which generate cavitation bubbles that collapse to produce high-energy jets, breaking up the layered crystallites culminating in exfoliated nanosheets with different lateral dimensions and thicknesses. ${ }^{9,23,26-28}$

In the exfoliation process, it has been shown that similarity in the surface energy and Hansen solubility parameters (HSP) of the solvent and the target material strongly affect the efficiency of the exfoliation. ${ }^{25,29-30}$ As a result, the choice of exfoliation solvent can play an important role in directing the plasmonic properties of 2D molybdenum oxide nanoflakes. Moreover, different HSP and surface energy components can alter the lateral dimensions and thickness of the flakes as well as the exfoliation yield, ${ }^{10,26}$ and hence can vary the plasmon dispersion relation. ${ }^{4,31-33}$ On the other hand, the change of solvent affects the water photooxidation process and potentially the degree of $\mathrm{H}^{+}$ion intercalation in 2D molybdenum oxide nanoflakes upon solar light illumination.

In mixtures of water and protic solvents, the oxidation reaction of the selected solvents competes ${ }^{34}$ with the water oxidation reaction, ${ }^{22}$ causing a reduced degree of $\mathrm{H}^{+}$intercalation 
and lower sub-stoichiometric levels in 2D molybdenum oxide nanoflakes. However, in mixtures of water and aprotic solvents, the oxidation reaction in selective solvents is

relatively limited, ${ }^{35-36}$ where the water oxidation reaction and sub-stoichiometric levels in 2D molybdenum oxide nanoflakes are in high degrees. This can generate higher free-electron concentrations and push the plasmonic resonance peak locations into the visible light region. ${ }^{4}$

In this study, we synthesized 2D molybdenum oxide nanoflakes via LPE in five mixtures of water with either aprotic or protic solvents. The samples were exposed to solar light for a fixed duration $(2 \mathrm{~h})$ and power intensity. Also, we characterized the plasmonic properties in relation to the samples' morphological parameters and free electron concentrations. The plasmonic biosensing performances were also investigated, in which bovine serum albumin (BSA) was used as a representative protein target.

\section{EXPERIMENTAL SECTION}

Synthesis of $2 \mathrm{D}$ Molybdenum oxide nanoflakes: $\mathrm{MoO}_{3}$ powder (99\% purity, China Rare Metal Material Co.) was ground with five different solvents, specifically N-methyl-2pyrrolidone (NMP), acetonitrile, methanol, ethanol and isopropyl alcohol (IPA), respectively. The grinding ratio was $3 \mathrm{~g}$ of the power in $0.6 \mathrm{~mL}$ solvent and the duration was $30 \mathrm{~min}$. The five samples were then dispersed in $45 \mathrm{~mL}$ of NMP/water, acetonitrile/water, methanol/water, ethanol/water and IPA/water mixtures $(50: 50 \mathrm{v} / \mathrm{v})$ respectively, and subjected to probesonication (Ultrasonic Processor Qsonica Q500) for $120 \mathrm{~min}$ at $125 \mathrm{~W}$. Subsequently, they were centrifuged at $3,220 \times \mathrm{g}$ for $30 \mathrm{~min}$ at room temperature. The five supernatant samples containing high concentrations of $2 \mathrm{D} \mathrm{MoO}_{3}$ flakes were collected and transferred into small glass vials of $10 \mathrm{~mL}$ volume each, which were then irradiated under a solar simulator (Abet Technologies LS-150) for $2 \mathrm{~h}$. 
Characterization: Digital Instruments D3100 atomic force microscopy (AFM) was used to investigate the surface morphology of drop-casted nanoflakes. Raman spectra were obtained micro-Raman spectrometer (Renishaw InVia microscope) with a 1200 lines per mm blazed grating and a $1 \mathrm{~mW}$ laser excitation source at $514 \mathrm{~nm}$. The baseline of Raman spectra were corrected by using Matlab (The Math Works, Natick MA, USA). ${ }^{37}$ Acquisitions were carried out for $10 \mathrm{~s}$ with two averages using a $\times 20$ magnification lens. The nanoflake suspensions were diluted and their average lateral dimensions were measured using dynamic light scattering (DLS) technique (ALV 5022F spectrometer). The X-ray diffraction (XRD) patterns were collected using a Bruker D4 ENDEAVOR with monochromatic $\mathrm{Cu} \mathrm{K} \alpha$ as radiation source $(\lambda=0.154 \mathrm{~nm})$. X-ray photoelectron spectroscopy (XPS) was performed using a Thermo scientific K-Alpha instrument equipped with a monochromated aluminium K- $\alpha$ source $(1486.7 \mathrm{eV})$. A pass energy of $20 \mathrm{eV}$ was selected and the binding energy of the Mo2p peaks was shifted so that the adventitious C1s peak was aligned to $284.8 \mathrm{eV}$. The supernatant containing 2D molybdenum oxide flakes were dropped onto $\mathrm{Cu}$ grid sample holders for high resolution transmission electron microcopy (HRTEM, using a JEOL 2100F) characterization. HRTEM images were acquired using a Gatan Orius SC1000 CCD Camera. Electron energy loss spectroscopy (EELS) was performed using a Gatan Imaging Filter (GIF) Tridium Spectrometer. To conduct EELS experiments, a high-angle annular dark field (HAADF)scanning transmission electron microscopy (STEM) image of the region of interest was first obtained. A small area map was then acquired across the nanoflakes, and the total number of data points was determined such that one spectrum was collected at every $2 \mathrm{~nm}$ interval. The zero loss peak (ZLP) of each spectrum was aligned using a digital micrograph and removed using the reflected tail model. Spectra within small areas of the map were then summed to improve the signal to noise ratio. The photoelectron spectroscopy in air (PESA) measurements were conducted on drop-casted samples on glass slides and were measured 
using a Riken Keiki Model AC-2 PESA spectrometer with power setting of $100 \mathrm{nW}$ and plotted using a power number of $1 / 3$, appropriate for semiconducting materials. Absorbance spectra were measured using an UV-Vis-NIR microspectrophotometer (CRAIC 20/30 PV). Zeta potentials of the mixtures were determined using a Zetasizer Nano ZS equipment (Malvern Instruments, Worcestershire, UK).

Thermogravimetric analysis (TGA) preparation: $24 \mathrm{~mL}$ of each suspension of 2D molybdenum oxide flakes was transferred onto 24 small centrifuge tubes of $1 \mathrm{~mL}$. The 24 tubes were centrifuged at very high speed of $21,000 \times \mathrm{g}$ for $60 \mathrm{~min}$ (Labogene-1524). A complete process is described in detail in the Supporting Information.

BSA preparation: To assess the biosensing capability of 2D molybdenum oxide flakes, $2 \mathrm{~mL}$ of BSA solutions (Invitrogen) with concentrations of $0.25,2.5,5$ and $10 \mathrm{mg} \mathrm{mL}^{-1}$ were incubated with $1 \mathrm{~mL}$ solutions of the irradiated suspended containing 2D molybdenum oxide nanoflakes for $1 \mathrm{~h}$ in different mixtures.

\section{RESULTS AND DISCUSSIONS}

3.1 Morphological characterizations of 2D molybdenum oxide nanoflakes. The lateral dimensions and thicknesses of 2D molybdenum oxide nanoflakes before (initial) and after $2 \mathrm{~h}$ exposure to solar illumination in different solvent/water mixtures were investigated using TEM, DLS and AFM. Figure 1a shows TEM images of 2D molybdenum oxide flakes in different mixtures before and after $2 \mathrm{~h}$ of solar illumination. Importantly, we can see different surface morphologies and shapes of the nanoflakes with various dimensions in different mixtures. ${ }^{32}$ The average lateral dimensions before and after solar illumination (Figure 1b) are extracted from the statistical analysis of the flake size distribution obtained from both DLS and TEM measurements (Figures S1 and S2). We observe a decrease 
trend in size following the sample order of NMP/water, acetonitrile/water, methanol/water, ethanol/water and IPA/water (Table S1). The largest average lateral dimension of the initial molybdenum oxide nanoflakes is found to be $\sim 450 \mathrm{~nm}$ for the NMP/water mixture, while the smallest is $200 \mathrm{~nm}$ in the IPA/water mixture. However, after $2 \mathrm{~h}$ of solar light illumination, the lateral dimensions of the flakes are reduced in both NMP/water and IPA/water mixtures to $\sim 380$ and $\sim 80 \mathrm{~nm}$, respectively.

a

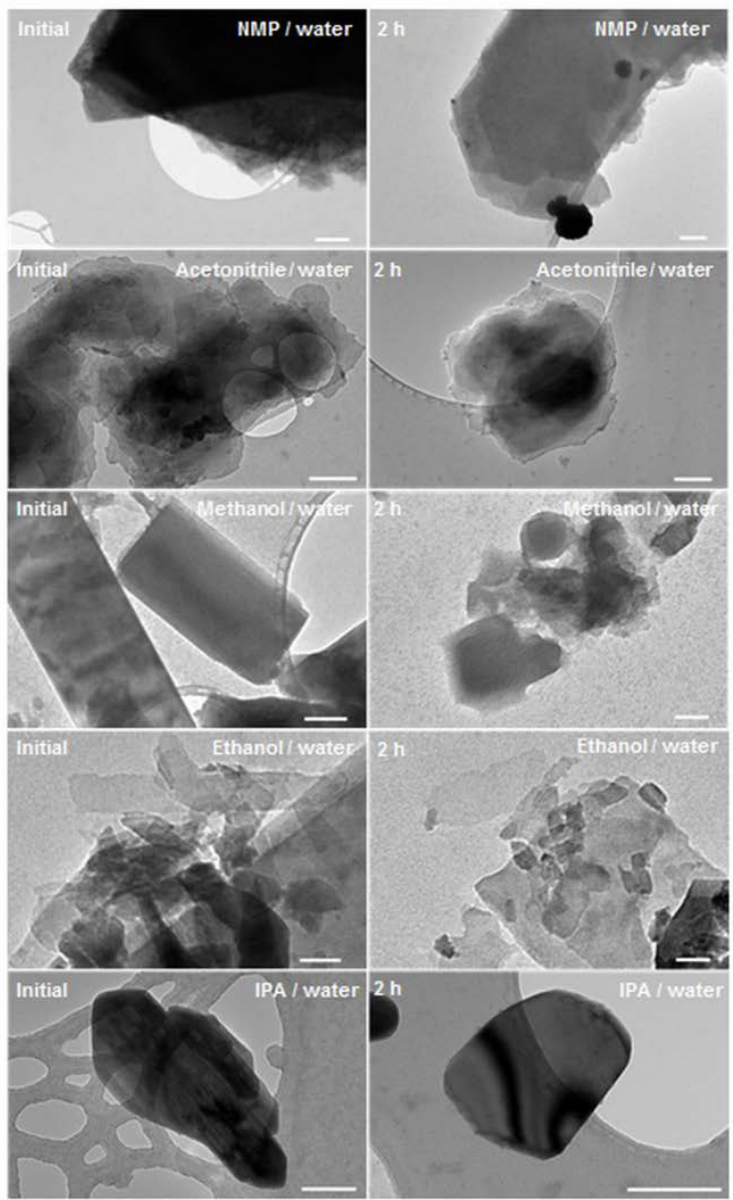

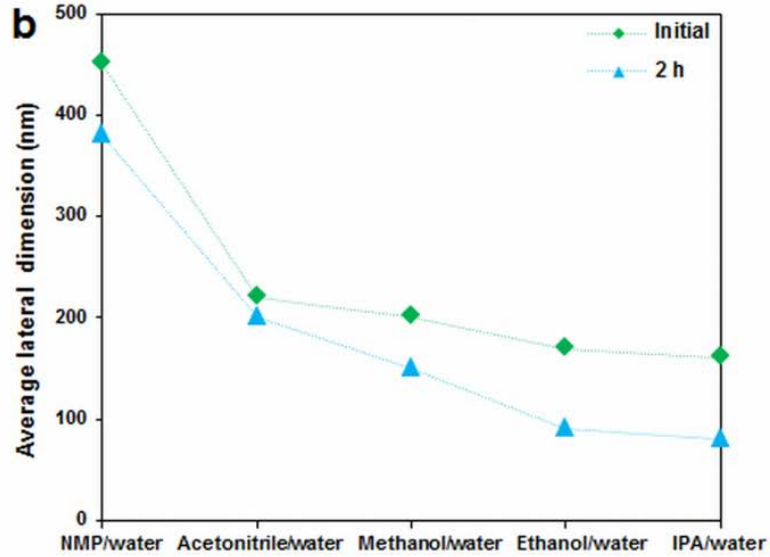

Solvent/water mixtures

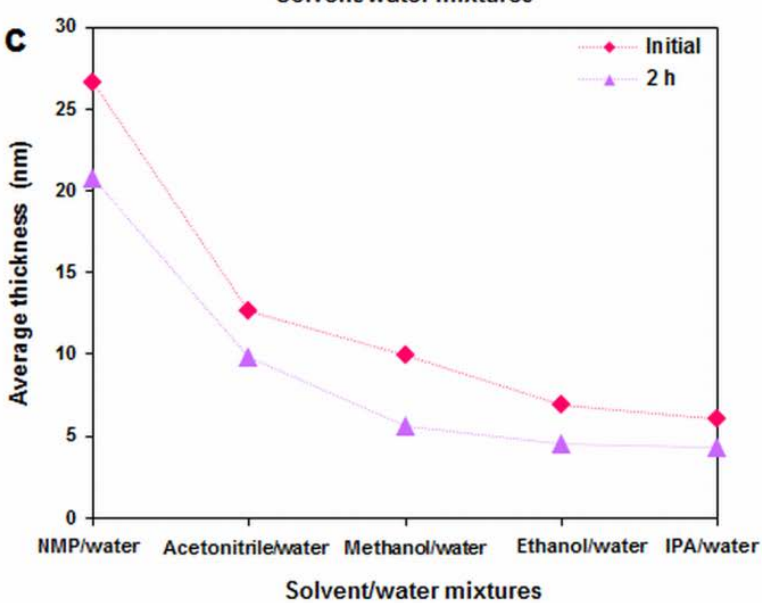

Figure 1. (a) TEM images of the initial nanoflakes and after solar illumination in different solvent/water mixtures (scale bars: $50 \mathrm{~nm}$ ). (b) The average lateral dimensions of 2D molybdenum oxide nanoflakes measured by DLS and TEM before and after $2 \mathrm{~h}$ solar illumination in different solvent mixtures. (c) The average thickness of the 2D nanoflakes before and after solar illumination in different mixtures obtained using AFM analysis.

AFM analysis confirmed the formation of 2D structure regardless of the choice of exfoliation solvent (Figures S3 and S4). Figure 1c shows the average AFM thickness of the 
molybdenum oxide flakes exfoliated in different solvent mixtures before and after solar illumination extracted from the statistical analysis of the flake thickness distribution shown in Figures S3 and S4. Notably, this follows a similar trend to the observed changes to the lateral dimension (Table S1). The largest average thickness of the initial molybdenum oxide nanoflakes is found to be 26 nm in NMP/water mixtures, while the smallest is $\sim 7 \mathrm{~nm}$ in IPA/water. However, after the solar illumination, the average thicknesses of these flakes are reduced to $\sim 20$ and $\sim 4 \mathrm{~nm}$, respectively. ${ }^{22}$ This is due to the photo-enhanced $\mathrm{H}^{+}$intercalation, leading to further exfoliation. ${ }^{22}$ Moreover, the rapid process of $\mathrm{H}^{+}$intercalation in the presence of solar light causes the formation of $\mathrm{OH}_{2}$ groups and then oxygen vacancies in molybdenum oxide nanoflakes, ${ }^{22}$ which results in crystal deformation and cracking in both intra-layer and inter-layer directions ${ }^{38}$ hence reducing the lateral dimension and thickness of the flakes. ${ }^{22}$

As the exfoliated material is $2 \mathrm{D}$, with a lateral dimension in the order of hundreds of nanometers, the interaction between the solvent and nanoflakes is assumed to primarily occur at the basal plane surface. ${ }^{39}$ A model to describe the balance of van der Waals intercalations is based on the enthalpy of nanoflake dispersion mix $\left(\Delta H_{m i x}\right)$ in the solvent per volume of the mixture $\left(V_{\text {mix }}\right)$ as follows: $:^{26,39}$

$$
\mathrm{T}_{\mathrm{f}} \approx \frac{2 V_{\text {mix }}}{\Delta H_{\text {mix }}} \times\left(\sqrt{\gamma_{s}}-\sqrt{\gamma_{\mathrm{f}}}\right)^{2} \times \phi
$$

where $T_{\mathrm{f}}$ is the nanoflake thickness, $\phi$ is the dispersed nanoflake volume fraction, and $\gamma_{\mathrm{s}}$ and $\gamma_{\mathrm{f}}$ are the total surface energies of solvent and nanoflakes, respectively.

The surface energies of the initial solvent/water mixtures are presented in Table S2, and their values are all higher than the surface energy of $\mathrm{MoO}_{3}\left(54-62 \mathrm{~mJ} / \mathrm{m}^{2}\right) .{ }^{40}$ From equation (1), we expect a minimal energy cost of exfoliation for solvent/water mixtures whose surface energy close to $\mathrm{MoO}_{3}{ }^{26}$ In addition, the thickness of the nanoflakes is quadratically dependent on the 
difference between surface energy of the solvent and $\mathrm{MoO}_{3}$ (Figure 1c). As predicted, the lateral dimension and thickness of the exfoliated nanoflakes are reduced concomitantly with a lowering of the surface energies of the mixtures in the order of NMP/water, acetonitrile/water, methanol/water, ethanol/water, and IPA/water, where the closest surface energy to $\mathrm{MoO}_{3}$ is found to be in IPA/water mixtures corresponding to smallest size and thickness.

On the other hand, the similarity in HSP parameters of the mixtures and molybdenum oxide can be related to the exfoliation yield of the nanoflakes. The dispersion of nanoflakes in liquid mixtures can be partially predicted by the HSP theory, ${ }^{39,41}$ which is a semi-empirical equation to present the dissolution behavior. ${ }^{39,} 41$ The interaction distance $\left(R_{\mathrm{a}}\right)$ between Hansen parameters is used for evaluating the level of adaptation between the suspension and the solvent as: $:^{41-42}$

$$
R_{\mathrm{a}}=\sqrt{\left[4 \times\left(\delta_{\mathrm{DS}}-\delta_{\mathrm{DF}}\right)^{2}+\left(\delta_{\mathrm{PS}}-\delta_{\mathrm{PF}}\right)^{2}+\left(\delta_{\mathrm{HS}}-\delta_{\mathrm{HF}}\right)^{2}\right]}
$$

Three HSP parameters are used for describing the character of a solvent $\left(\delta_{\mathrm{S}}\right)$ and a flake material $\left(\delta_{\mathrm{F}}\right)$ with the respect to dispersive $\left(\delta_{\mathrm{D}}\right)$, polar $\left(\delta_{\mathrm{P}}\right)$, and hydrogen-bonding $\left(\delta_{\mathrm{H}}\right)$ solubility parameters. The smaller the $R_{\mathrm{a}}$ value leads to higher expected solubility and hence larger production yield. Also, the concentration $(C)$ of $2 \mathrm{D}$ materials dispersed in the mixture is maximized when the solubility parameters for the mixtures and 2D material match, which can be approximated by: ${ }^{30,39}$

$$
C \propto \exp \left[-\frac{v_{\mathrm{F}}}{3 k T}\left(\delta_{\mathrm{TS}}-\delta_{\mathrm{TF}}\right)^{2}\right]
$$

where $\delta_{\mathrm{TS}}$ and $\delta_{\mathrm{TF}}$ represents the Hildebrand solubility parameters of solvent and $\mathrm{MoO}_{3}$ flakes, and $v_{\mathrm{F}}$ represents the molecular volume of the $\mathrm{MoO}_{3}$ flakes. For $2 \mathrm{D} \mathrm{MoO}_{3}$, its $\delta_{\mathrm{TF}}$ is 
estimated to be $20.7 \mathrm{MPa}^{1 / 2}$ from similar examples. ${ }^{30}$ Its HSP parameters are $\delta_{\mathrm{DF}}=17.8$ $\mathrm{MPa}^{1 / 2}, \delta_{\mathrm{PF}}=8 \mathrm{MPa}^{1 / 2}$, and $\delta_{\mathrm{HF}}=6.5 \mathrm{MPa}^{1 / 2} \cdot 30,39$

From equations (2) and (3), the trend for $R_{\mathrm{a}}$ and $\left(\delta_{\mathrm{TS}}-\delta_{\mathrm{TF}}\right)$ values are extracted from HSP and Hildebrand solubility parameters to confirm that it follows the order of NMP/water, acetonitrile/water, IPA/water, ethanol/water, and methanol/water, respectively (Table S3). Notably, this indicates that the highest production yield of $2 \mathrm{D} \mathrm{MoO}_{3}$ nanoflakes is realized for the NMP/water mixture, while the lowest production yield is obtained from the methanol/water mixture.

This is experimentally validated using thermogravimetric analysis (TGA) to determine the exfoliation yield. ${ }^{22}$ Measuring the yield of exfoliated material is not trivial, since classical methods can lead to incorrect estimations. Photometric analysis is not accurate due to size dependent spectral changes of $\mathrm{MoO}_{3}$ nanoflakes. Wet chemical analysis and inductively coupled plasma mass spectrometry (ICP-MS) or atomic absorption spectroscopy can also result in misleading values, since both $\mathrm{MoO}_{3}$ nanoflakes and dissolved molybdic acid are present in the suspensions and cannot be distinguished by those methods. Finally, gravimetric techniques are impacted by the large amount of surface bound solvent molecules, which can constitute up to $70 \%$ of the dried sample. ${ }^{28}$ Quantitative gravimetric analysis using TGA can overcome this limitation and lead to a more accurate estimation of the yield (see the process in Note 1 and Figures S5 and S6). The yields of the $2 \mathrm{D} \mathrm{MoO}_{3}$ flakes are measured as $\sim 0.033$, $\sim 0.022, \sim 0.018, \sim 0.006$, and $\sim 0.004 \mathrm{mg} \mathrm{mL}^{-1}$ in NMP/water, IPA/water, ethanol/water, and methanol/water, and acetonitrile/water mixtures, respectively. The experimental trend (Figure S6) matches the calculated parameters (Table S2) except for the case of the acetonitrile/water mixture. This is perhaps due to the high affinity of acetonitrile for water molecules, resulting 
in an unexpected increase of the $R_{\mathrm{a}}$ value as well as the HSP and Hildebrand solubility parameters and hence a slightly lower than expected production yield. ${ }^{43}$

The colloidal stabilities of 2D $\mathrm{MoO}_{3}$ nanoflakes in different solvent mixtures are evaluated using zeta potential measurements. From Table S4, the zeta potential values for all mixtures are negative, in which the most negative is found in IPA/water mixture (-95.5 mV) while the least lowest is in $\mathrm{NMP} /$ water mixture $(-7.54 \mathrm{mV})$. As the larger absolute zeta potential value indicates more electrostatically stability of the suspension, ${ }^{44}$ it is implied that the IPA/water mixture may be the most stable and NMP/water mixture is the least. Their long term stabilities will be discussed in detail in the later sections.

3.2 Structural characterizations of 2D molybdenum oxide nanoflakes. XRD was utilized to assess the crystal structure of $2 \mathrm{D}$ molybdenum oxide flakes before and after solar irradiation in different mixtures (Figure S7). The XRD patterns show that the initial 2D molybdenum oxide nanoflakes are made of crystalline orthorhombic $\mathrm{Mo}_{17} \mathrm{O}_{47}$ in the NMP/water sample, while others are made of orthorhombic $\alpha-\mathrm{MoO}_{3}$ (Figure S7). ${ }^{45}$ This observation was also confirmed by HRTEM and SAED patterns (Figure S8). However, after solar light illumination, some of the lattice fringes appear disordered after the solar illumination as a consequence of defect and oxygen vacancies generation upon the $\mathrm{H}^{+}$ intercalation, which confirmed the presence of polycrystalline ring SAED pattern in all mixtures (Figure S8). ${ }^{3,22}$

The Raman spectra of the molybdenum oxide flakes in different mixtures, before and after solar illumination, were also investigated (Figure 2a,c,e,g,i). Strong Raman peaks in the initial samples are observed at 280, 335, 666, 820 and $994 \mathrm{~cm}^{-1}$ in the ethanol/water sample (Figure $2 \mathrm{~g}$ ), which are all in good agreement with orthorhombic $\alpha-\mathrm{MoO}_{3}$ phase (Note 2, Supporting Information). ${ }^{4}{ }^{24}$ For the acetonitrile/water, methanol/water and IPA/water samples (Figure 2c,e,i), an additional peak is observed at $962 \mathrm{~cm}^{-1}$ which can be assigned to 
$\mathrm{Mo}=\mathrm{O}$ stretching mode of slightly sub-stoichiometric $\mathrm{MoO}_{3-\mathrm{x} \cdot}{ }^{4,}$, ${ }^{46}$ For the NMP/water sample (Figure 2a), traces of $\mathrm{MoO}_{3-x}$ are also identified by two additional peaks at 440 and $750 \mathrm{~cm}^{-1}$ due to the modification of the original $\mathrm{Mo}_{2}-\mathrm{O}$ bond. ${ }^{4,}$ 6, 24, 46 From Raman analysis, the existence of some sub-stoichiometry in the initial 2D molybdenum oxide nanoflakes can be possibly due to the intercalation of $\mathrm{H}^{+}$from the solvent in the presence of significant heat and force generated during the sonication process, which can eventually create oxygen vacancies. $^{27,47-48}$ The highest sub-stoichiometry is found in NMP/water sample, possibly due to the fact that NMP is a known oxygen scavenger, ${ }^{49-50}$ which contributes to increased oxygen removal compared to all other solvent mixtures evaluated in this study. ${ }^{49-50}$

After exposure to solar light, the dominant Raman peaks appearing at 236, 483, 720 and $820 \mathrm{~cm}^{-1}$ correspond to the formation of $\mathrm{MoO}_{3-x},{ }^{24}$ while the intensity of the $\alpha-\mathrm{MoO}_{3}$ Raman peaks gradually decrease in all samples (Figure 2a,c,e,g,i). The peaks at $236 \mathrm{~cm}^{-1}$ can be assigned to the doubly coordinated oxygen $\mathrm{Mo}_{2}-\mathrm{O}$ bending mode of $\mathrm{MoO}_{3-x}$, while the peaks at 483, 720 and $820 \mathrm{~cm}^{-1}$ can be assigned to $\mathrm{Mo}_{3}-\mathrm{O}, \mathrm{Mo}_{2}-\mathrm{O}$ and $\mathrm{Mo}=\mathrm{O}$ stretching modes, respectively. ${ }^{24,46}$

In addition to the Raman spectroscopy, XPS analysis was also conducted to further validate the sub-stoichiometry of the 2D nanoflakes before and after solar light illumination (Figure 2b,d,f,h,j).The XPS analysis survey shows that there is no existence of impurities or organic species on the surface of the initial samples were observed from the residue of a liquid-based organic solvent-assisted grinding and sonication method (Figure S9). Moreover, the observed doublets in each of the initial samples centered at 232.58 and $235.48 \mathrm{eV}$ are characteristic of fully-stoichiometric $\mathrm{MoO}_{3}$ and may be attributed to higher oxidation states of $\mathrm{Mo}^{6+} 3 \mathrm{~d}_{5 / 2}$ and $\mathrm{Mo}^{6+} 3 \mathrm{~d}_{3 / 2}$, respectively (Figure 2b,d,f,h,j). ${ }^{4,6,23}$ However, after the solar irradiation, doublet peaks centered at $231.38 \mathrm{eV}$ for $\mathrm{Mo}^{5+} 3 \mathrm{~d}_{5 / 2}$ and $234.38 \mathrm{eV}$ for $\mathrm{Mo}^{5+} 3 \mathrm{~d}_{3 / 2}$ are detected for all samples, indicating the presence of $\mathrm{MoO}_{3-x}$ (Figure 2b,d,f,h,j). ${ }^{4,6}$ 

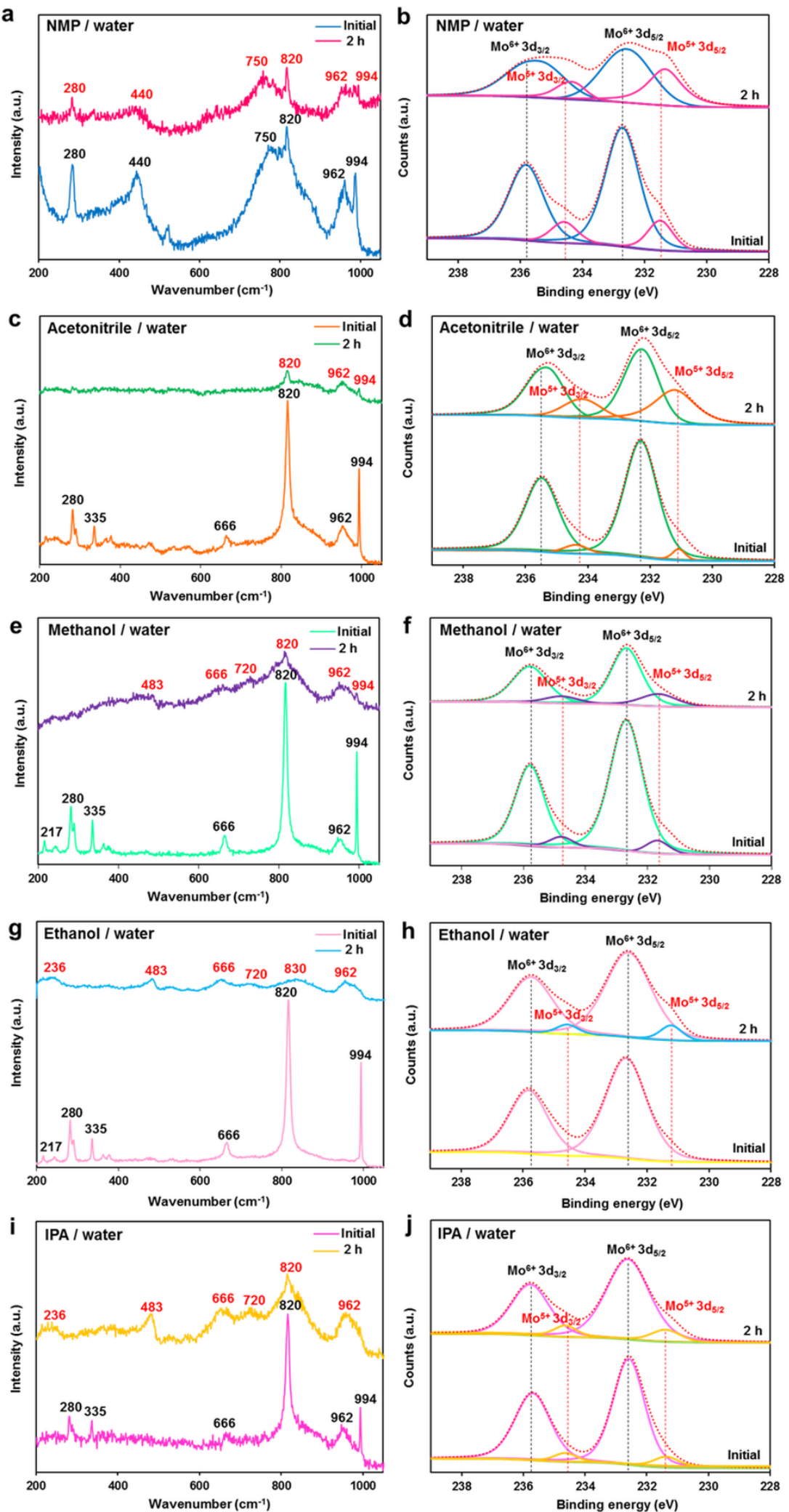

Figure 2. The Raman spectra of the $2 \mathrm{D}$ molybdenum oxide flakes before and after solar irradiation in (a) NMP/water, (c) acetonitrile/water, (e) methanol/water, (g) ethanol/water and (i) IPA/water mixtures. The corresponding XPS Mo3d spectra before and after solar irradiation in (b) NMP/water, (d) acetonitrile/water, (f) methanol/water, (h) ethanol/water and (j) IPA/water mixtures. 
Figure 3a shows the electronic band structure of 2D molybdenum oxide flakes in different mixtures constructed using a combination of PESA, Tauc plot analysis, and valence photoemission spectroscopy both before and after solar light illumination (Note 3 in Supporting Information, Figures S10, S11 and S12, and Table S5). In contrast to the initial sample, solar light irradiation shifts the Fermi level $\left(E_{F}\right)$ upward towards the conduction band in all samples, which indicates the increase in the doping level arising from oxygen vacancies. $^{22,51}$ Furthermore, the bandgap energy $\left(E_{g}\right)$ is slightly reduced in all samples (Figure 3a). Interestingly, the $E_{F}$ of $2 \mathrm{D}$ molybdenum oxide flakes in the NMP/water sample is higher than the conduction band minimum, which indicates its metallic properties are stronger than the other samples. Moreover, we believe because of the photo-excitation, the solvent oxidation is competing with the water oxidation, leading to reduced $\mathrm{H}^{+}$intercalation. In order to explain the solvent oxidation mechanism, we classify the solvents in two groups: protic solvents, in which the molecules of the solvent are hydrogen bonded to each other including methanol, ethanol, IPA, and aprotic solvents, in which the molecules of the solvent are not hydrogen bonded to each other including NMP and acetonitrile. ${ }^{52}$ In protic solvents, IPA is the more easily oxidized than ethanol and methanol, which implies that water oxidation and $\mathrm{H}^{+}$intercalation are of lower degrees for IPA in comparison to ethanol and methanol. ${ }^{53}$ This is because of the activation energy $\left(E_{\mathrm{a}}\right)($ IPA $<$ ethanol $<$ methanol), wherein the lowest $E_{\mathrm{a}}$ value shows the best electro-oxidation activity. ${ }^{34}$ However, aprotic solvents such as NMP and acetonitrile are of limited strength for oxidation and water molecules are also less firmly bonded to those solvents. ${ }^{35-36}$ Moreover, NMP has the highest proton affinity $\left(\sim 920 \mathrm{~kJ} \mathrm{~mol}^{-1}\right)^{54-56}$ and smallest proton dissociation lifetime. ${ }^{55-57}$ This leads to a higher degree of $\mathrm{H}^{+}$intercalation process in NMP than acetonitrile. 

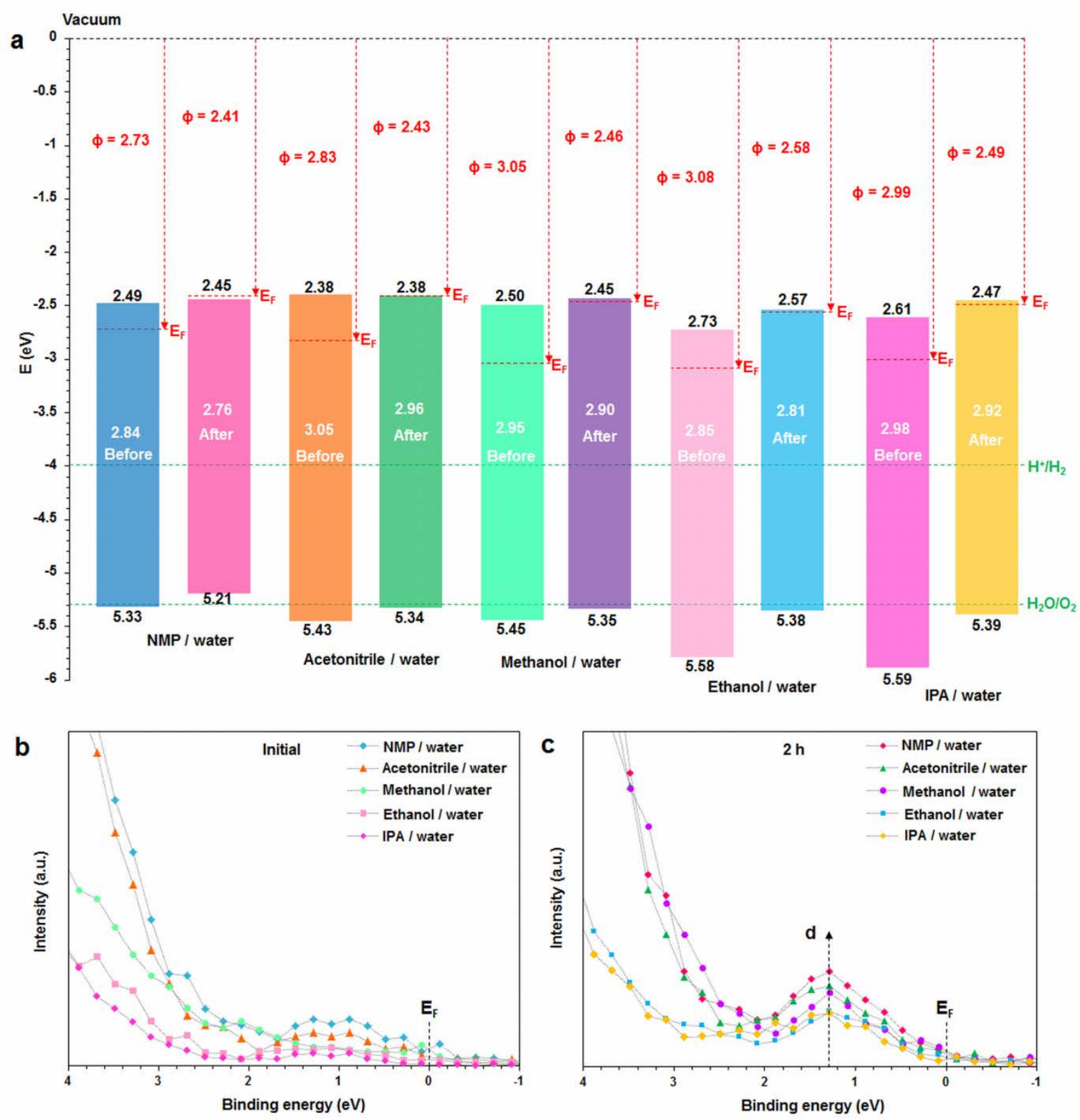

Figure 3. (a) The assessed electronic band structure values of 2D molybdenum oxide samples before and after solar illumination in different mixtures. (b) The valence photoemission spectroscopy of the initial 2D molybdenum oxide samples in different mixtures. (c) The substoichiometric molybdenum oxide valence photoemission spectroscopy patterns in different mixtures after solar illumination.

The free electron concentrations $(N)$ per unit volume $\left(\mathrm{cm}^{-3}\right)$ for different samples after solar light illumination are estimated from the $\mathrm{Mo}^{5+} / \mathrm{Mo}^{6+}$ area ratio in $\mathrm{MoO}_{3-x}$, which are extracted from the XPS measurements (Table 1). It is found that $N$ increases for all samples upon solar light irradiation as the oxygen vacancies increase. ${ }^{58}$ Figure $3 b$ shows the valence 
photoemission spectra of sub-stoichiometric 2D molybdenum oxides at low binding energies before solar irradiation for different samples. However, after the solar light irradiation (introducing oxygen vacancies) a new occupied state appears within the $E_{g}$ of $\mathrm{MoO}_{3-\chi}$, labeled $d$ in Figure 3c. ${ }^{22}$ This defect state arises because an $\mathrm{O}^{2-}$ ion is removed from the valence-band of $\mathrm{MoO}_{3-x}{ }^{22,} 58$ The previously empty 4d band of $\mathrm{MoO}_{3}$ becomes partially occupied with electrons, giving rise to the defect band (1.28 eV below $\left.E_{F}\right)$ which is enhanced in intensity by increasing the solar light irradiation duration (Figure 3c). ${ }^{22,} 58$ More importantly, such a defect band represents shallow donor states which donate free electrons into molybdenum oxide nanoflakes, ${ }^{58-60}$ resulting in the enhancement of free electron concentration of the nanoflakes and theoretically blue-shifting the plasmon resonance peaks in both thickness and lateral dimension axes of a 2D plasmonic system (detailed discussion will be presented in the later sections).

TABLE 1. Estimated sub-stoichiometry, free electron concentrations and phases of 2D molybdenum oxide flakes for different mixtures upon $2 \mathrm{~h}$ of solar light irradiation.

\begin{tabular}{ccccc}
\hline Mixtures & $\begin{array}{c}\text { XPS area ratio of } \\
\left(\mathrm{Mo}^{5+} / \mathrm{Mo}^{6+}\right.\end{array}$ & $\begin{array}{c}\text { Sub-stoichiometric }(x) \\
\text { of } \mathrm{MoO}_{3-x}\end{array}$ & $\begin{array}{c}\text { Estimated free electron } \\
\text { concentration }(N)\left(\mathrm{cm}^{-3}\right)\end{array}$ & Phases \\
\hline NMP/water & 0.470 & 0.240 & $9.8 \times 10^{21}$ & $\mathrm{MoO}_{2.75}$ \\
Acetonitrile/water & 0.375 & 0.187 & $7.35 \times 10^{21}$ & $\mathrm{MoO}_{2.80}$ \\
Methanol/water & 0.250 & 0.125 & $3.92 \times 10^{21}$ & $\mathrm{MoO}_{2.875}$ \\
Ethanol/water & 0.167 & 0.084 & $3.28 \times 10^{21}$ & $\mathrm{MoO}_{2.90}$ \\
IPA/water & 0.125 & 0.063 & $2.46 \times 10^{21}$ & $\mathrm{MoO}_{2.937}$ \\
\hline
\end{tabular}

3.3 Plasmonic properties of 2D molybdenum oxide nanoflakes. The optical properties of the molybdenum oxide nanoflakes were investigated using UV-Vis-NIR spectroscopy. The 
UV-Vis-NIR spectra of the initial samples are shown in Figure S13, with no obvious absorption peak in the visible light region for pristine $2 \mathrm{D} \mathrm{MoO}_{3}$ flakes. However, after solar illumination (Figure 4 and Table S6), two broad absorption plasmonic peaks of different intensities appear in all mixtures.

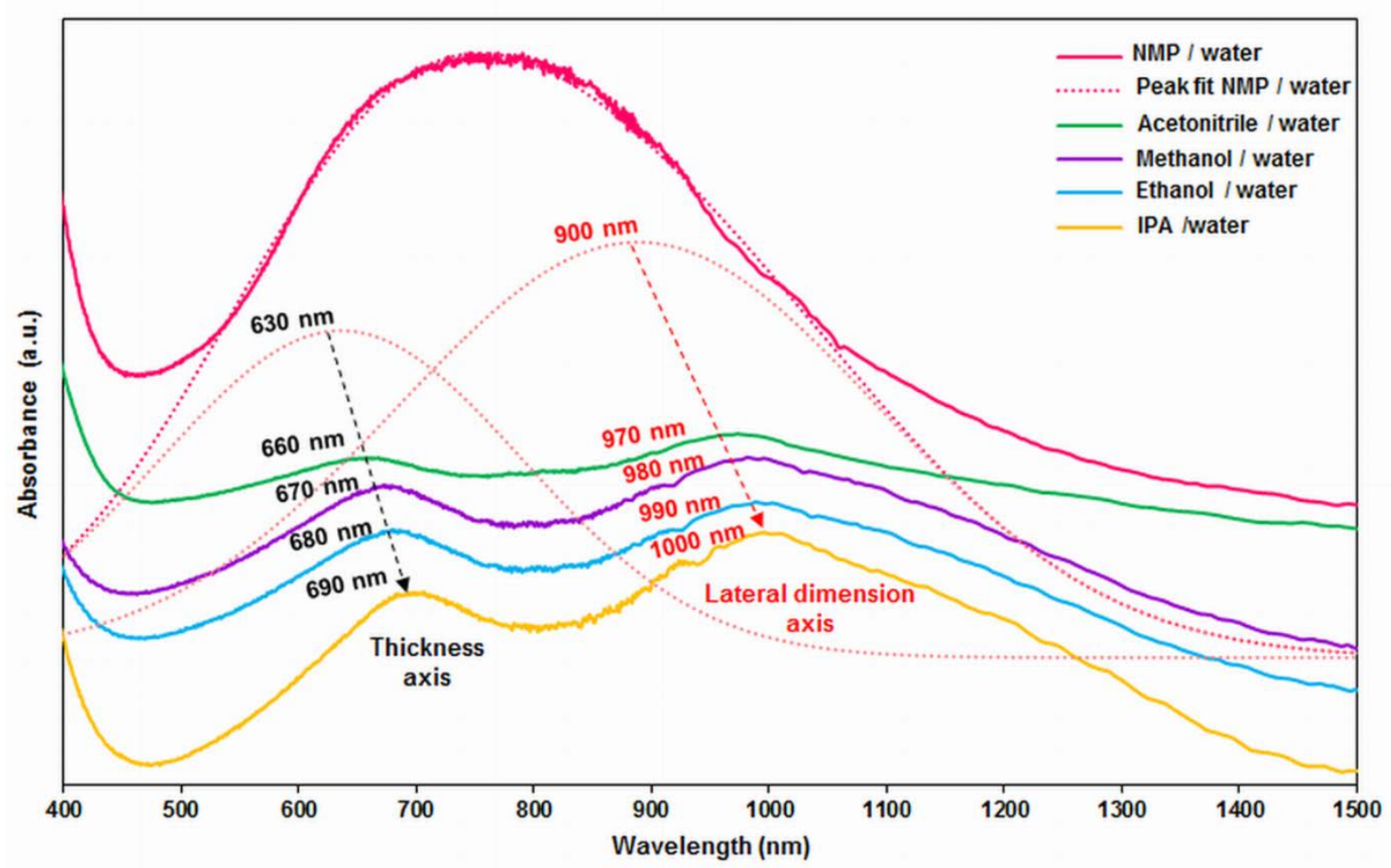

Figure 4. The UV-Vis-NIR absorbance spectra of the samples after solar light illumination in different solvent/water mixtures.

For the IPA/water sample, a low intensity absorption peak is centered at $690 \mathrm{~nm}$, while a high intensity peak is centered at $1000 \mathrm{~nm}$. Remarkably, the intensities of those two absorption plasmon peaks decrease in the ethanol/water sample and are blue shifted to 680 and $990 \mathrm{~nm}$, respectively. Moreover, a continuous decreasing trend in the intensities of these two peaks together with a blue shifting of $\sim 10 \mathrm{~nm}$ in the methanol/water and acetonitrile/water samples are seen. However in the NMP/water sample, we can only observe one broad absorption peak with a relatively higher intensity in comparison to all other samples. This broad peak can be 
fitted using Gaussian peak-fitting into two peaks centered at 630 and $900 \mathrm{~nm}$, matching the previous blue shifting trend of the peaks (Figure 4). The existence of the absorption plasmon peaks confirms our Mie-Gans calculations (Note 4 in Supporting Information and Tables S7). The plasmon absorption peak response is known to be aspect ratio-dependent. ${ }^{4,33,61-62} \mathrm{We}$ associate the peak with the low intensity ranged between 630 and 690 to the thickness (thickness axis) and the peak of the higher intensity $(900-1000 \mathrm{~nm})$ to the lateral dimension (lateral dimension axis). Nevertheless, according to Mie-Gans calculations, the variation in the plasmonic peaks with respect to the average aspect ratios of $\mathrm{MoO}_{3-x}$ nanoflakes is almost the same for all samples. In this case, this can be due to strong dependency on the free electron concentration rather than aspect ratio of the flakes, in which the absorbance spectrum of the material (NMP/water) with larger electron concentration is blue-shifted compared to that with lower free electron concentration (IPA/water). ${ }^{61,63}$

The extinction coefficients associated with both axes are high $\left(\varepsilon>10^{9} \mathrm{~L} \cdot \mathrm{mol}^{-1} \cdot \mathrm{cm}^{-1}\right)$ for all solar light irradiated samples as shown in Table 2. The calculation of the extinction coefficients is described in details by the equations presented in Note 5 and Table S8 in Supporting Information.

TABLE 2. The extinction coefficients of the thickness and lateral dimension axes of the samples in different mixtures after solar light irradiation.

\begin{tabular}{|c|c|c|c|c|}
\hline Mixtures & $\lambda_{\max }$ at the thickness axis (nm) & $\varepsilon_{t}\left(\mathrm{~L} \cdot \mathrm{mol}^{-1} \cdot \mathrm{cm}^{-1}\right)$ & $\lambda_{\max }$ at the lateral dimension axis (nm) & $\varepsilon_{l}\left(\mathrm{~L} \cdot \mathrm{mol}^{-1} \cdot \mathrm{cm}^{-1}\right)$ \\
\hline NMP/water & 630 & $1.90 \times 10^{11}$ & 900 & $2.45 \times 10^{11}$ \\
\hline Acetonitrile/water & 660 & $3.54 \times 10^{10}$ & 970 & $5.10 \times 10^{10}$ \\
\hline Methanol/water & 670 & $1.77 \times 10^{10}$ & 980 & $2.25 \times 10^{10}$ \\
\hline Ethanol/water & 680 & $2.29 \times 10^{9}$ & 990 & $2.65 \times 10^{9}$ \\
\hline IPA/water & 690 & $1.85 \times 10^{9}$ & 1000 & $2.34 \times 10^{9}$ \\
\hline
\end{tabular}


The long term stabilities of $2 \mathrm{D} \mathrm{MoO}_{3-x}$ suspension in different solvent mixtures after 3 month storage at room temperature were investigated using Raman and UV-Vis-NIR spectroscopy. As shown in Figure S14, there is no significant alteration of Raman and absorbance spectra observed in ethanol/water and IPA/water mixtures. However, for the acetonitrile/water and methanol/water mixtures, their sub-stoichiometry are significantly lower as their Raman peaks at $\sim 820 \mathrm{~cm}^{-1}$ related to full stoichiometry are enhanced and both plasmonic peaks exhibit red-shifts, indicating their oxygen vacancies are recombined over time. For the NMP/water sample, its plasmonic absorption peak is obviously narrowed while there is no significant difference can be found in its Raman peaks related to substoichiometry, possibly indicating its aspect ratio (lateral dimension over thickness in a 2D system) is altered over time. This is confirmed by the optical image (Figure S15) and AFM analysis (Figure S16), in which significant aggregation of the nanoflakes is observed possibly due to the relatively electrostatic instability in NMP/water sample (see the zeta potential values presented in Table S4).

The relationship between the free electron concentration and the plasmonic resonance peak position was also confirmed by EELS. ${ }^{6,64-65}$ Figure 5a-j shows the EELS spectra collected from the samples of 2D molybdenum oxides corresponding to STEM images before and after solar light illumination. A Gaussian peak-fitting technique is employed to extract the plasmon and the interband transition modes from the spectra shown in Figure 5a-j. For the initial 2D molybdenum oxide (Figure 5a,c,e,g,i), deconvoluted peaks centered at 5.4 eV (acetonitrile/water), $5.7 \mathrm{eV}$ (ethanol/water), $6.4 \mathrm{eV}$ (methanol/water) and $6.5 \mathrm{eV}$ (NMP/water and IPA/water) appear which can be ascribed to interband transitions. ${ }^{6,} 66$ Prominent peaks centered between 12.2-15.8 and 24.5-26.8 eV are observed, which can be attributed to the 
plasmon modes induced by valence electrons in outer atomic cells. ${ }^{6}$ Also, a broad peak centered at 36.5-37.7 eV and $49.6 \mathrm{eV}$ can be assigned to the core-loss transition from Mo-4p excitation. ${ }^{6}$ An additional strong peak appears only after solar illumination for all samples that centered at $7.5 \mathrm{eV}$ (NMP/water), $6.7 \mathrm{eV}$ (acetonitrile/water), $5.7 \mathrm{eV}$ (methanol/water), $4.4 \mathrm{eV}$ (ethanol/water) and $4 \mathrm{eV}$ (IPA/water), which can be attributed to the bulk plasmon resonance originating from the generated free electrons in the presence of oxygen vacancies (Figure 5b,d,f,h,j and Table S9).

This can be further validated by the theoretical calculations for the bulk plasmon peak energies using the free electron gas model, expressed as follows: ${ }^{6}$

$$
E_{p}=\hbar \sqrt{\frac{N e^{2}}{\varepsilon_{o} m_{e}}}
$$

where $\hbar$ is the reduced Planck constant, the free electron concentration $(N)$ is presented in Table $1, e$ is the elementary charge, $\varepsilon_{\mathrm{o}}$ is the permittivity of free space and $m_{\mathrm{e}}$ is the effective mass of electrons (Note 4, Supporting Information). The highest value of the bulk plasmon peak mode position of 2D molybdenum oxide flakes is seen in the NMP/water sample, which correlates to the highest free electron concentration and strongest sub-stoichiometric level $(x)$, while the lowest value is noted from the IPA/water sample, which correlates to the lowest free electron concentration (fewer oxygen vacancies) (Figure S17). 

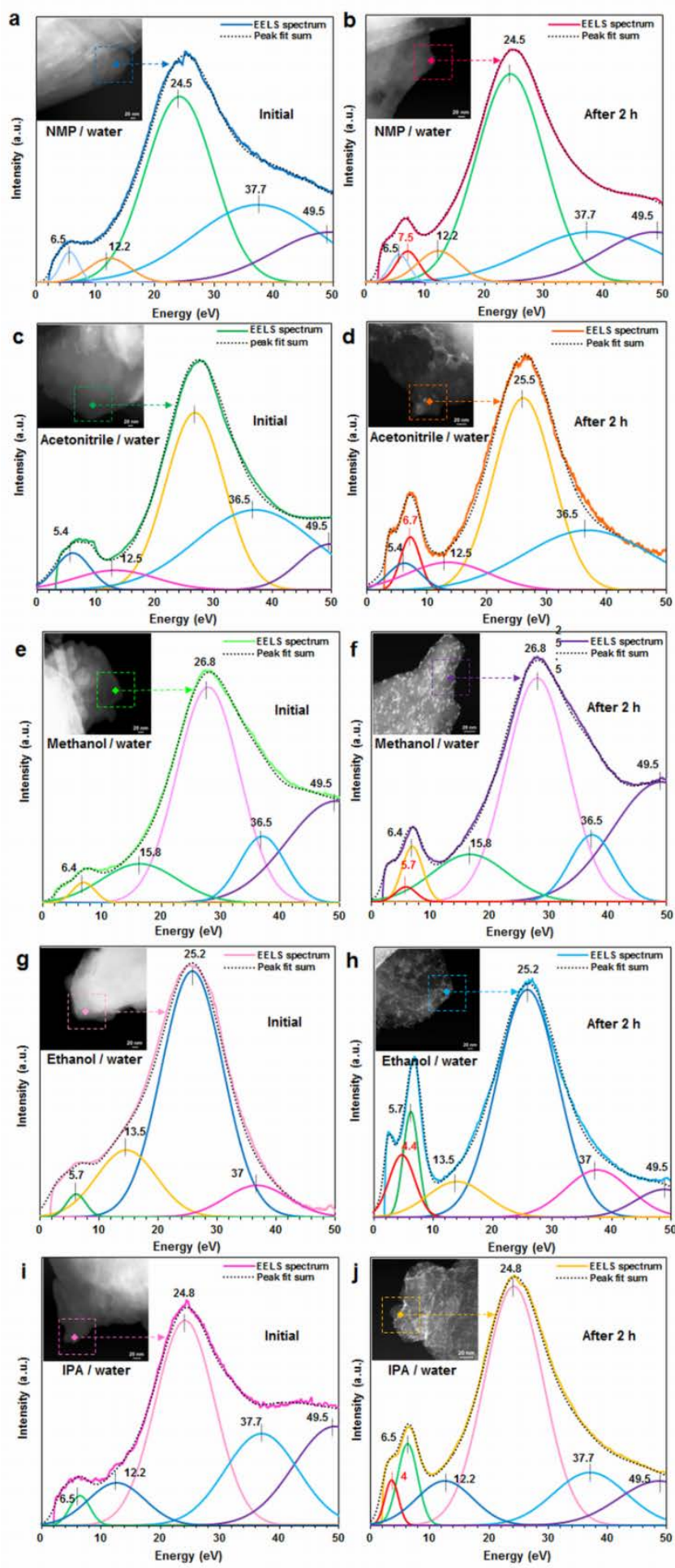

Figure 5. The EELS spectra of the initial 2D nanoflakes in (a) NMP/water, (c) acetonitrile/water, (e) methanol/water, (g) ethanol/water and (i) IPA/water mixtures with the corresponding STEM images in inset. The EELS spectra of the 2D nanoflakes after solar light illumination in (b) NMP/water, (d) acetonitrile/water, (f) methanol/water, (h) ethanol/water and (j) IPA/water mixtures with corresponding STEM images in inset. The plural scattering (multiple plasmon resonances) were removed in these graphs to eliminate the thickness effect. 


\subsection{Plasmonic biosensing application.}

In order to further explore the applicability of our 2D plasmonic molybdenum oxide flakes, an optical biosensing system was devised in which BSA was used as a model protein for assessing the sensitivity. We have previously shown that BSA had a very high affinity to molybdenum oxide and was readily immobilized onto the surface of the flakes. ${ }^{4}$ Suspended sub-stoichiometric 2D flakes in different mixtures were used in these experiments. BSA immobilization on the oxide surface is achieved via collective van der Waals' forces and electrostatic interactions between the protein functional groups and the surface of flakes. ${ }^{4,67-}$ 68 The negatively charged immobilized BSA repels the free electrons present at the nanoflakes surface. ${ }^{4}$ This leads to a decrease in the free electron density near the surface of the flakes, hence altering the plasmonic properties of 2D molybdenum oxide flakes. ${ }^{4}$ As observed in Figure S14, upon the exposure to BSA of the concentration of $0.25 \mathrm{mg} \mathrm{mL}^{-1}$, the intensities of thickness-axis plasmonic peaks are reduced for all the samples but no obvious peak shift is seen. Figure 6a represents the response factor trend in different mixtures, and the response factor is defined as the ratio of $2 \mathrm{D}$ molybdenum oxide plasmonic peak intensity before BSA exposure over the peak intensity after BSA exposure. The highest response factor is found in the IPA/water sample that has the lowest free electron concentration and smallest flake size, while the lowest response factor is in NMP/water sample that correlates to the highest free electron concentration and largest flake size (Table S10). This is rationalized since the smaller sized nanoflakes produces a more confined electromagnetic field, making it more sensitive to BSA molecules. ${ }^{33}$ Upon the increase of BSA concentration (Figure $6 \mathrm{~b}$ and Figure S18), both the NMP/water and acetonitrile/water mixtures have a linear increase of the BSA response factors for up to $10 \mathrm{mg} \mathrm{mL}^{-1}$. For other mixtures, the linear responses increase at higher rates but become non-linear beyond the concentration of $5 \mathrm{mg} \mathrm{mL}^{-1}$. 

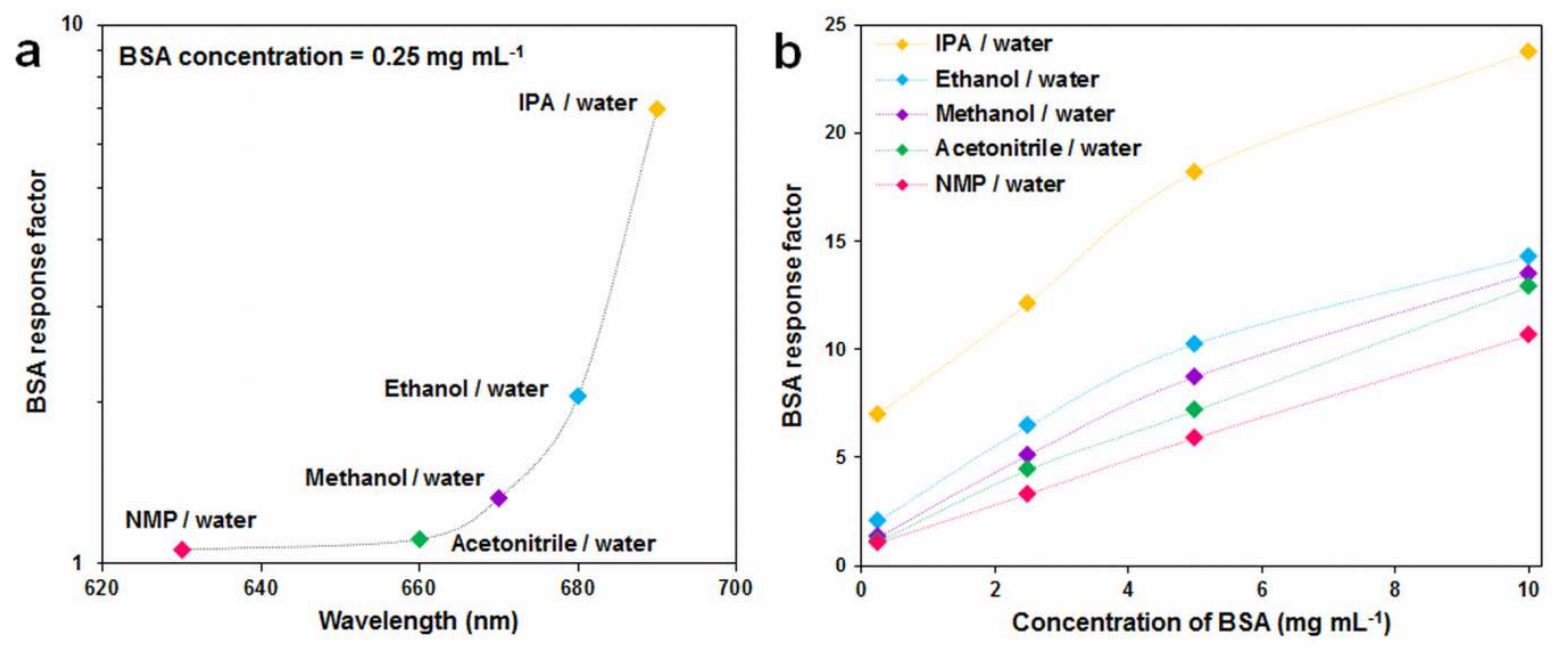

Figure 6. (a) BSA response factors of the 2D molybdenum oxide biosensors based on different mixtures corresponding to thickness axis. BSA of $0.25 \mathrm{mg} \mathrm{mL}^{-1}$ is used in the measurements. (b) The corresponding BSA response factors of the biosensor as a function of the BSA concentration $\left(0.25,2.5,5\right.$ and $\left.10 \mathrm{mg} \mathrm{mL}^{-1}\right)$. The BSA response factors values are extracted from Figure S18.

It is seen that the reduction in the intensity of the lateral-dimension-axis plasmonic peaks upon the BSA exposure are much more significant than those of thickness-axis related peaks for all samples since the lateral-dimension-axis plasmonic peaks here nearly disappear. This observation is similar to the plasmonic biosensing performances observed in one-dimension gold nanorods, where the plasmon absorption peak corresponding to longitudinal band of the nanorods is more sensitive to surface adsorptive changes than that of the transverse band. ${ }^{69-70}$ The structural stability of 2D $\mathrm{MoO}_{3-x}$ nanoflakes after BSA exposure was investigated by the Raman spectroscopy. Figure S19 reveals that after the exposure of BSA, the main Mo-O stretching Raman peaks of sub-stoichiometric molybdenum oxide in the region between 600 and $1000 \mathrm{~cm}^{-1}$ mostly remain for all mixtures, although there are significantly broaden possibly due to the organic interference from BSA. This indicates that the crystal structure of the 2D nanoflakes is not significantly altered upon the exposure of bio-spices. 


\section{CONCLUSIONS}

In liquid phase exfoliation of 2D molybdenum oxide flakes, we showed the surface energy and solubility of solvent/water mixtures played important roles in modifying the thickness, lateral dimension and production yield. Upon photo-excitation of the exfoliated 2D molybdenum oxide samples by simulated solar light, water oxidation and $\mathrm{H}^{+}$intercalation of the flakes occurred. In protic solvents, solvent oxidation competition for water oxidation led to a reduced degree of the photo intercalation process. However, in aprotic solvents, the solvent oxidation reaction was relatively limited and the water oxidation reaction and photo $\mathrm{H}^{+}$intercalation occurred with higher degrees. Increase in proton intercalation in 2D molybdenum oxide nanoflakes resulted in a significant increase in their free electron concentrations and change in their sub-stoichiometry. We observed that two plasmon resonance peaks emerged for each sample. These plasmon resonance peaks of the 2D molybdenum oxide nanoflakes were tunable in the visible range, depending on the choice of the exfoliation solvent. The plasmonic peak shifts were strongly dependent on free electron concentrations in the nanoflakes rather than their thickness and lateral dimensions. Additionally, 2D molybdenum oxide nanoflakes in all mixtures showed very high extinction coefficients in the order of $\varepsilon>10^{9} \mathrm{~L} \cdot \mathrm{mol}^{-1} \cdot \mathrm{cm}^{-1}$. Finally, biosensing capability of plasmonic 2D molybdenum oxide nanoflake samples was demonstrated using BSA as a model protein. Altogether, the process presented in this paper was introduced as an efficient method for producing and tuning 2D flakes with plasmonic properties that will have future applications in many sensing and optical platforms. 


\section{ASSOCIATED CONTENT}

\section{Supporting Information}

The content of Supporting Information includes the following: DLS, TEM, AFM and HRTEM characterizations, lateral dimension and thickness histograms, XRD patterns, detailed analysis of the TGA process, surface energies calculations, HSP and Hildebrand solubility calculations, Zeta potential and PESA measurements, Tauc plots, energy level diagrams and the valence photoemission spectra of 2D molybdenum oxides flakes for different mixtures, UV-Vis-NIR absorbance spectra for the initial 2D molybdenum oxide nanoflakes in different mixtures, optical image and AFM image of 2D molybdenum oxide flakes after 3 month of the same fresh sample of NMP/water mixtures, the extinction coefficient and theoretical calculations for plasmon resonance peaks, and BSA response factor based on 2D molybdenum oxide flakes in different mixtures.

\section{AUTHOR INFORMATION}

\section{Corresponding Author}

E-mail: manalmyaalsaif@gmail.com

E-mail: jianzhen.ou@rmit.edu.au

E-mail: kourosh.kalantar@rmit.edu.au

\section{Notes}

The authors declare no competing financial interest.

\section{ACKNOWLEDGMENTS}

The authors would like to acknowledge funding support from the Australian Research Council (ARC) through Discovery Project DP140100170 and the DECRA project DE160100715. The authors would also like to acknowledge the facilities, scientific and technical assistances of the Australian Microscopy and Microanalysis Research Facility 
(AMMRF) at RMIT University, the Micro Nano Research Facility (MNRF) at RMIT University and the Australian Centre for Materials Science and Engineering (CMSE) of the Commonwealth Scientific and Industrial Research Organization (CSIRO), Australia.

\section{REFERENCES}

(1) Garcia de Abajo, F. J. Graphene Plasmonics: Challenges and Opportunities. ACS Photonics 2014, 1, 135-152.

(2) Naik, G. V.; Shalaev, V. M.; Boltasseva, A. Alternative Plasmonic Materials: Beyond Gold and Silver. Adv. Mater. 2013, 25, 3264-3294.

(3) Wang, Y.; Ou, J. Z.; Chrimes, A. F.; Carey, B. J.; Daeneke, T.; Alsaif, M. M. Y. A.; Mortazavi, M.; Zhuiykov, S.; Medhekar, N.; Bhaskaran, M.; Friend, J. R.; Strano, M. S.; Kalantar-Zadeh, K. Plasmon Resonances of Highly Doped Two-Dimensional $\mathrm{MoS}_{2}$. Nano Lett. 2015, 15, 883-890.

(4) Alsaif, M. M. Y. A.; Latham, K.; Field, M. R.; Yao, D. D.; Medehkar, N. V.; Beane, G. A.; Kaner, R. B.; Russo, S. P.; Ou, J. Z.; Kalantar-zadeh, K. Tunable Plasmon Resonances in Two-Dimensional Molybdenum Oxide Nanoflakes. Adv. Mater. 2014, 26, 3931-3937.

(5) Gordon, T. R.; Paik, T.; Klein, D. R.; Naik, G. V.; Caglayan, H.; Boltasseva, A.; Murray, C. B. Shape-Dependent Plasmonic Response and Directed Self-Assembly in a New Semiconductor Building Block, Indium-Doped Cadmium Oxide (ICO). Nano Lett. 2013, 13, 2857-2863.

(6) Alsaif, M. M. Y. A.; Field, M. R.; Murdoch, B. J.; Daeneke, T.; Latham, K.; Chrimes, A. F.; Zoolfakar, A. S.; Russo, S. P.; Ou, J. Z.; Kalantar-zadeh, K. Substoichiometric TwoDimensional Molybdenum Oxide Flakes: A Plasmonic Gas Sensing Platform. Nanoscale 2014, 6, 12780-12791. 
(7) Ju, L.; Geng, B.; Horng, J.; Girit, C.; Martin, M.; Hao, Z.; Bechtel, H. A.; Liang, X.; Zettl, A.; Shen, Y. R.; Wang, F. Graphene Plasmonics for Tunable Terahertz Metamaterials. Nat. Nanotechnol. 2011, 6, 630-634.

(8) Cheng, H.; Qian, X.; Kuwahara, Y.; Mori, K.; Yamashita, H. A Plasmonic Molybdenum Oxide Hybrid with Reversible Tunability for Visible-Light-Enhanced Catalytic Reactions. Adv. Mater. 2015, 27, 4616-4621.

(9) Nicolosi, V.; Chhowalla, M.; Kanatzidis, M. G.; Strano, M. S.; Coleman, J. N. Liquid Exfoliation of Layered Materials. Science 2013, 340, 6139.

(10) Varrla, E.; Backes, C.; Paton, K. R.; Harvey, A.; Gholamvand, Z.; McCauley, J.; Coleman, J. N. Large-Scale Production of Size-Controlled $\mathrm{MoS}_{2}$ Nanosheets by Shear Exfoliation. Chem. Mater. 2015, 27, 1129-1139.

(11) Wang, Q. H.; Kalantar-Zadeh, K.; Kis, A.; Coleman, J. N.; Strano, M. S. Electronics and Optoelectronics of Two-Dimensional Transition Metal Dichalcogenides. Nat. Nanotechnol. 2012, 7, 699-712.

(12) Balendhran, S.; Walia, S.; Nili, H.; Ou, J. Z.; Zhuiykov, S.; Kaner, R. B.; Sriram, S.; Bhaskaran, M.; Kalantar-zadeh, K. Two-Dimensional Molybdenum Trioxide and Dichalcogenides. Adv. Funct. Mater. 2013, 23, 3952-3970.

(13) Xia, F.; Wang, H.; Xiao, D.; Dubey, M.; Ramasubramaniam, A. Two-Dimensional Material Nanophotonics. Nat. Photonics 2014, 8, 899-907.

(14) Scholz, A.; Stauber, T.; Schliemann, J. Plasmons and Screening in a Monolayer of MoS $_{2}$. Phys. Rev. B 2013, 88, 035135.

(15) Luo, X.; Qiu, T.; Lu, W.; Ni, Z. Plasmons in Graphene: Recent Progress and Applications. Mater. Sci. Eng., R 2013, 74, 351-376.

(16) Zhao, H.; Guo, Q.; Xia, F.; Wang, H. Two-Dimensional Materials for Nanophotonics Application. Nanophotonics 2015, 4, 128-142. 
(17) Andersen, K.; Thygesen, K. S. Plasmons in Metallic Monolayer and Bilayer Transition Metal Dichalcogenides. Phys. Rev. B 2013, 88, 155128.

(18) Noguez, C. Surface Plasmons on Metal Nanoparticles: The Influence of Shape and Physical Environment. J. Phys. Chem. C 2007, 111, 3806-3819.

(19) Hu, M.; Chen, J.; Li, Z.-Y.; Au, L.; Hartland, G. V.; Li, X.; Marquez, M.; Xia, Y. Gold Nanostructures: Engineering Their Plasmonic Properties for Biomedical Applications. Chem. Soc. Rev. 2006, 35, 1084-1094.

(20) Wang, H.; Yuan, H.; Hong, S. S.; Li, Y.; Cui, Y. Physical and Chemical Tuning of TwoDimensional Transition Metal Dichalcogenides. Chem. Soc. Rev. 2015, 44, 2664-2680.

(21) Yan, H.; Li, X.; Chandra, B.; Tulevski, G.; Wu, Y.; Freitag, M.; Zhu, W.; Avouris, P.; Xia, F. Tunable Infrared Plasmonic Devices Using Graphene/Insulator Stacks. Nat. Nanotechnol. 2012, 7, 330-334.

(22) Alsaif, M. M. Y. A.; Chrimes, A. F.; Daeneke, T.; Balendhran, S.; Bellisario, D. O.; Son, Y.; Field, M. R.; Zhang, W.; Nili, H.; Nguyen, E. P.; Latham, K.; Embden, J. v.; Strano, M. S.; Ou, J. Z.; Kalantar-zadeh, K. High-Performance Field Effect Transistors Using Electronic Inks of 2D Molybdenum Oxide Nanoflakes. Adv. Funct. Mater. 2015, DOI: 10.1002/adfm.201503698.

(23) Alsaif, M. M. Y. A.; Balendhran, S.; Field, M. R.; Latham, K.; Wlodarski, W.; Ou, J. Z.; Kalantar-zadeh, K. Two Dimensional $\alpha-\mathrm{MoO}_{3}$ Nanoflakes Obtained Using Solvent-Assisted Grinding and Sonication Method: Application for $\mathrm{H}_{2}$ Gas Sensing. Sens. Actuators, B 2014, 192, 196-204.

(24) Ou, J. Z.; Carnpbell, J. L.; Yao, D.; Wlodarski, W.; Kalantar-zadeh, K. In Situ Raman Spectroscopy of $\mathrm{H}_{2}$ Gas Interaction with Layered $\mathrm{MoO}_{3}$. J. Phys. Chem. C 2011, 115, 1075710763. 
(25) Shen, J.; He, Y.; Wu, J.; Gao, C.; Keyshar, K.; Zhang, X.; Yang, Y.; Ye, M.; Vajtai, R.; Lou, J.; Ajayan, P. M. Liquid Phase Exfoliation of Two-Dimensional Materials by Directly Probing and Matching Surface Tension Components. Nano Lett. 2015, 15, 5449-5454.

(26) Hernandez, Y.; Nicolosi, V.; Lotya, M.; Blighe, F. M.; Sun, Z.; De, S.; McGovern, I. T.; Holland, B.; Byrne, M.; Gun'ko, Y. K.; Boland, J. J.; Niraj, P.; Duesberg, G.; Krishnamurthy, S.; Goodhue, R.; Hutchison, J.; Scardaci, V.; Ferrari, A. C.; Coleman, J. N. High-Yield Production of Graphene by Liquid-Phase Exfoliation of Graphite. Nat. Nanotechnol. 2008, 3, 563-568.

(27) Qiao, W.; Yan, S.; He, X.; Song, X.; Li, Z.; Zhang, X.; Zhong, W.; Du, Y. Effects of Ultrasonic Cavitation Intensity on the Efficient Liquid-Exfoliation of $\mathrm{MoS}_{2}$ Nanosheets. RSC Adv. 2014, 4, 50981-50987.

(28) Carey, B. J.; Daeneke, T.; Nguyen, E. P.; Wang, Y.; Ou, J. Z.; Zhuiykov, S.; KalantarZadeh, K. Two Solvent Grinding Sonication Method for the Synthesis of Two-Dimensional Tungsten Disulphide Flakes. Chem. Commun. 2015, 51, 3770-3773.

(29) Coleman, J. N.; Lotya, M.; O'Neill, A.; Bergin, S. D.; King, P. J.; Khan, U.; Young, K.; Gaucher, A.; De, S.; Smith, R. J.; Shvets, I. V.; Arora, S. K.; Stanton, G.; Kim, H.-Y.; Lee, K.; Kim, G. T.; Duesberg, G. S.; Hallam, T.; Boland, J. J.; Wang, J. J.; Donegan, J. F.; Grunlan, J. C.; Moriarty, G.; Shmeliov, A.; Nicholls, R. J.; Perkins, J. M.; Grieveson, E. M.; Theuwissen, K.; McComb, D. W.; Nellist, P. D.; Nicolosi, V. Two-Dimensional Nanosheets Produced by Liquid Exfoliation of Layered Materials. Science 2011, 331, 568-571.

(30) Hanlon, D.; Backes, C.; Higgins, T. M.; Hughes, M.; O’Neill, A.; King, P.; McEvoy, N.; Duesberg, G. S.; Mendoza Sanchez, B.; Pettersson, H.; Nicolosi, V.; Coleman, J. N. Production of Molybdenum Trioxide Nanosheets by Liquid Exfoliation and Their Application in High-Performance Supercapacitors. Chem. Mater. 2014, 26, 1751-1763. 
(31) Rycenga, M.; Cobley, C. M.; Zeng, J.; Li, W.; Moran, C. H.; Zhang, Q.; Qin, D.; Xia, Y. Controlling the Synthesis and Assembly of Silver Nanostructures for Plasmonic Applications. Chem. Rev. 2011, 111, 3669-3712.

(32) Burda, C.; Chen, X. B.; Narayanan, R.; El-Sayed, M. A. Chemistry and Properties of Nanocrystals of Different Shapes. Chem. Rev. 2005, 105, 1025-1102.

(33) Anker, J. N.; Hall, W. P.; Lyandres, O.; Shah, N. C.; Zhao, J.; Van Duyne, R. P. Biosensing with Plasmonic Nanosensors. Nat. Mater. 2008, 7, 442-453.

(34) Wang, D.; Liu, J.; Wu, Z.; Zhang, J.; Su, Y.; Liu, Z.; Xu, C. Electrooxidation of Methanol, Ethanol and 1-Propanol on Pd Electrode in Alkaline Medium. Int. J. Electrochem. Sci 2009, 4, 1672-1678.

(35) Zoski, C. G. Handbook of Electrochemistry. Elsevier: Oxford, 2006.

(36) Hammerich, O.; Lund, H. Organic Electrochemistry. 4th ed.; Marcel Dekker: New York, 2001.

(37) Chrimes, A. F.; Khoshmanesh, K.; Tang, S.-Y.; Wood, B. R.; Stoddart, P. R.; Collins, S. S.; Mitchell, A.; Kalantar-Zadeh, K. In Situ SERS Probing of Nano-Silver Coated Individual Yeast Cells. Biosens. Bioelectron. 2013, 49, 536-541.

(38) Spahr, M. E.; Novak, P.; Haas, O.; Nesper, R. Electrochemical Insertion of Lithium, Sodium, and Magnesium in Molybdenum(Vi) Oxide. J. Power Sources 1995, 54, 346-351.

(39) Cunningham, G.; Lotya, M.; Cucinotta, C. S.; Sanvito, S.; Bergin, S. D.; Menzel, R.; Shaffer, M. S. P.; Coleman, J. N. Solvent Exfoliation of Transition Metal Dichalcogenides: Dispersibility of Exfoliated Nanosheets Varies Only Weakly between Compounds. ACS Nano 2012, 6, 3468-3480.

(40) Halim, U.; Zheng, C. R.; Chen, Y.; Lin, Z.; Jiang, S.; Cheng, R.; Huang, Y.; Duan, X. A Rational Design of Cosolvent Exfoliation of Layered Materials by Directly Probing LiquidSolid Interaction. Nat. Commun. 2013, 4, 2213-2219. 
(41) Hansen, C. M. Hansen Solubility Parameters: A User's Handbook. CRC press: LLC, Boca Raton, Florida, 2007.

(42) Zhou, K.-G.; Mao, N.-N.; Wang, H.-X.; Peng, Y.; Zhang, H.-L. A Mixed-Solvent Strategy for Efficient Exfoliation of Inorganic Graphene Analogues. Angew. Chem., Int. Ed. 2011, 50, 10839-10842.

(43) Williams, D. B. G.; Lawton, M. Drying of Organic Solvents: Quantitative Evaluation of the Efficiency of Several Desiccants. J. Org. Chem. 2010, 75, 8351-8354.

(44) Li, Y.; Zhu, H.; Shen, F.; Wan, J.; Lacey, S.; Fang, Z.; Dai, H.; Hu, L. Nanocellulose as Green Dispersant for Two-Dimensional Energy Materials. Nano Energy 2015, 13, 346-354.

(45) Wang, Z.; Madhavi, S.; Lou, X. W. Ultralong $\alpha-\mathrm{MoO}_{3}$ Nanobelts: Synthesis and Effect of Binder Choice on Their Lithium Storage Properties. J. Phys. Chem. C 2012, 116, 1250812513.

(46) Camacho-Lopez, M. A.; Escobar-Alarcon, L.; Picquart, M.; Arroyo, R.; Cordoba, G.; Haro-Poniatowski, E. Micro-Raman Study of the $\mathrm{m}-\mathrm{MoO}_{2}$ to $\alpha-\mathrm{MoO}_{3}$ Transformation Induced by CW-Laser Irradiation. Opt. Mater. 2011, 33, 480-484.

(47) Mao, M.; Wang, M.; Hu, J.; Lei, G.; Chen, S.; Liu, H. Simultaneous Electrochemical Synthesis of Few-Layer Graphene Flakes on Both Electrodes in Protic Ionic Liquids. Chem. Commun. 2013, 49, 5301-5303.

(48) Jing, Y.; Zhou, Z.; Cabrera, C. R.; Chen, Z. Graphene, Inorganic Graphene Analogs and Their Composites for Lithium Ion Batteries. J. Mater. Chem. A 2014, 2, 12104-12122.

(49) Dubin, S.; Gilje, S.; Wang, K.; Tung, V. C.; Cha, K.; Hall, A. S.; Farrar, J.; Varshneya, R.; Yang, Y.; Kaner, R. B. A One-Step, Solvothermal Reduction Method for Producing Reduced Graphene Oxide Dispersions in Organic Solvents. ACS Nano 2010, 4, 3845-3852. 
(50) White, C. M.; Rohar, P. C.; Veloski, G. A.; Anderson, R. R. Practical Notes on the Use of N-Methyl-2-Pyrrolidinone as a Solvent for Extraction of Coal and Coal-Related Materials. Energy Fuels 1997, 11, 1105-1106.

(51) Wang, Y.; Ou, J. Z.; Balendhran, S.; Chrimes, A. F.; Mortazavi, M.; Yao, D. D.; Field, M. R.; Latham, K.; Bansal, V.; Friend, J. R.; Zhuiykov, S.; Medhekar, N. V.; Strano, M. S.; Kalantar-zadeh, K. Electrochemical Control of Photoluminescence in Two-Dimensional $\mathrm{MoS}_{2}$ Nanoflakes. ACS Nano 2013, 7, 10083-10093.

(52) Marcus, Y. Ions in Solution and Their Solvation. Wiley: Hoboken, New Jersey, 2015.

(53) Xie, S.-W.; Chen, S.; Liu, Z.-Q.; Xu, C.-W. Comparison of Alcohol Electrooxidation on Pt and Pd Electrodes in Alkaline Medium. Int. J. Electrochem. Sci 2011, 6, 882-888.

(54) Webster, G. K. Supercritical Fluid Chromatography: Advances and Applications in Pharmaceutical Analysis. CRC Press: Boca Raton, Florida, 2014.

(55) Niessen, W. M. Liquid Chromatography-Mass Spectrometry. 3rd ed.; CRC Press: Boca Raton, Florida, 2006.

(56) Yu, F. E.; Hsu, J. M.; Pan, J. P.; Wang, T. H.; Chiang, Y. C.; Lin, W.; Jiang, J. C.; Chern, C. S. Effect of Solvent Proton Affinity on the Kinetics of Michael Addition Polymerization of N, N'-Bismaleimide-4, 4'-Diphenylmethane with Barbituric Acid. Polym. Eng. Sci. 2014, 54, 559-568.

(57) Pines, E.; Fleming, G. R. Proton Transfer in Mixed Water-Organic Solvent Solutions: Correlation between Rate, Equilibrium Constant, and the Proton Free Energy of Transfer. J. Phys. Chem. 1991, 95, 10448-10457.

(58) Greiner, M. T.; Chai, L.; Helander, M. G.; Tang, W.-M.; Lu, Z.-H. Transition Metal Oxide Work Functions: The Influence of Cation Oxidation State and Oxygen Vacancies. Adv. Funct. Mater. 2012, 22, 4557-4568. 
(59) Wang, G.; Ling, Y.; Wang, H.; Yang, X.; Wang, C.; Zhang, J. Z.; Li, Y. HydrogenTreated $\mathrm{WO}_{3}$ Nanoflakes Show Enhanced Photostability. Energy Environ. Sci. 2012, 5, 61806187.

(60) Yan, J.; Wang, T.; Wu, G.; Dai, W.; Guan, N.; Li, L.; Gong, J. Tungsten Oxide Single Crystal Nanosheets for Enhanced Multichannel Solar Light Harvesting. Adv. Mater. 2015, 27, $1580-1586$.

(61) Luther, J. M.; Jain, P. K.; Ewers, T.; Alivisatos, A. P. Localized Surface Plasmon Resonances Arising from Free Carriers in Doped Quantum Dots. Nat. Mater. 2011, 10, 361366.

(62) Manthiram, K.; Alivisatos, A. P. Tunable Localized Surface Plasmon Resonances in Tungsten Oxide Nanocrystals. J. Am. Chem. Soc. 2012, 134, 3995-3998.

(63) Agrawal, A.; Kriegel, I.; Milliron, D. J. Shape-Dependent Field Enhancement and Plasmon Resonance of Oxide Nanocrystals. J. Phys. Chem. C 2015, 119, 6227-6238.

(64) Koh, A. L.; Bao, K.; Khan, I.; Smith, W. E.; Kothleitner, G.; Nordlander, P.; Maier, S. A.; McComb, D. W. Electron Energy-Loss Spectroscopy (EELS) of Surface Plasmons in Single Silver Nanoparticles and Dimers: Influence of Beam Damage and Mapping of Dark Modes. ACS Nano 2009, 3, 3015-3022.

(65) Scholl, J. A.; Koh, A. L.; Dionne, J. A. Quantum Plasmon Resonances of Individual Metallic Nanoparticles. Nature 2012, 483, 421-427.

(66) Lajaunie, L.; Boucher, F.; Dessapt, R.; Moreau, P. Strong Anisotropic Influence of Local-Field Effects on the Dielectric Response of $\alpha-\mathrm{MoO}_{3}$. Phys. Rev. B 2013, 88, 115141.

(67) Balendhran, S.; Walia, S.; Alsaif, M.; Nguyen, E. P.; Ou, J. Z.; Zhuiykov, S.; Sriram, S.; Bhaskaran, M.; Kalantar-zadeh, K. Field Effect Biosensing Platform Based on 2D $\alpha-\mathrm{MoO}_{3}$. ACS Nano 2013, 7, 9753-9760. 
(68) Song, L.; Yang, K.; Jiang, W.; Du, P.; Xing, B. Adsorption of Bovine Serum Albumin on Nano and Bulk Oxide Particles in Deionized Water. Colloids Surf., B 2012, 94, 341-346.

(69) Cao, J.; Sun, T.; Grattan, K. T. V. Gold Nanorod-Based Localized Surface Plasmon Resonance Biosensors: A Review. Sens. Actuators, B 2014, 195, 332-351.

(70) Pérez-Juste, J.; Pastoriza-Santos, I.; Liz-Marzán, L. M.; Mulvaney, P. Gold Nanorods: Synthesis, Characterization and Applications. Coord. Chem. Rev. 2005, 249, 1870-1901. 


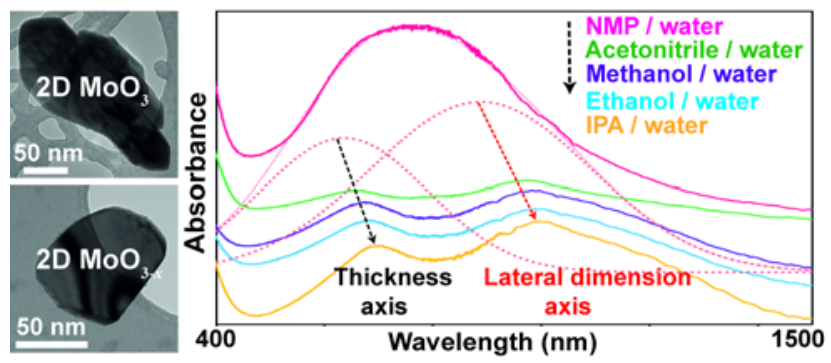

TOC Figure 